\title{
Retrospective Study of Demographic, Etiological Factors, Clinical Outcome and Postoperative Complications Encountered In Tendon Achilles Injury
}

\author{
Dr Rajalakshmi ${ }^{1}$.G, Dr Veeranna ${ }^{2}$, Dr Sushil kumar ${ }^{3}$, Dr Anitha Muthgi ${ }^{4}$, Dr \\ Nrupathunga K.K \\ ${ }^{1}$ Assistant professor/Plastic surgeon, Dept. Of Surgery \\ ${ }^{2}$ Associate professor, Dept. Of Orthopaedics \\ ${ }^{3}$ Professor and HOD, Dept. Of surgery \\ ${ }^{4,5}$ Intern, Dept. Of Surgery Shimoga Institute of Medical Sciences, Shimoga, Karnataka, India
}

\begin{abstract}
Injury to tendoachilles are common following sports, road traffic accidents, fall in Indian lavatory pan etc. Direct repair was done with prolene or stainless steel wires with primary closure of skin. Complications ranged from minor to deep tissue infection and persistent steel wire. This is a retrospective study of acute tendo Achilles injury, managed in SHIMOGA INSTITUTE OF MEDICAL SCIENCES during September 2012-13. It is a study of demographic, etiologic factors, clinical outcome and postoperative complications encountered.
\end{abstract}

Key words: Achilles tendon, acute tendo Achilles injury, tendoachilles rupture

1.1 History

\section{Introduction}

Achilles, Greek warrior and hero of Homer's Iliad and the Trojan War. Dipped in the river Styx by his mother Thetis to make him invincible, he was however left vulnerable at the part by which she held him which was the heel. Although not told in the Iliad, he is said to have been killed by a poisoned arrow to the heel shot by Paris, the brother of the Trojan prince Hector.

Hence came the term Achilles heel, which is defined as a deadly weakness in spite of overall strength. The term was first used by VERHEYDEN, Dutch anatomist; he dissected his own ${ }^{5}$ amputated leg in $1963^{5}$.

Hippocrates, the father of western medicine states-"this tendon, if bruised or cut, causes the most acute fevers induces choking, deranges the mind and at length brings death"

Guglielmo di Falcieto (12 ${ }^{\text {th }}$ century) advocated operative repair of acute Achilles tendon rupture. Ambroise Pare (1575) recommended that ruptured Achilles tendon be strapped with bandages dipped in wine. It was however Gustave Polaillon (1888) who popularised operative repair.

\subsection{Anatomy ${ }^{4}$}

The Achilles tendon is the largest tendon in the body which comprises of the tendinous portions of the gastrocnemius and soleus muscle that inserts into the calcaneus. Distally, the tendon becomes progressively rounded and at about $4 \mathrm{~cm}$ proximal to insertion becomes flatter.

At its insertion $2-5 \mathrm{~cm}$ distal to the tip of superior calcaneal tuberosity, the fibres of the gastrocnemius lie laterally and those of the soleus lie medially. The fibres spiral towards their insertion on calcaneal tuberosity. Its adjacent soft tissue relations include kagers fat pad anteriorly and retro Achilles bursa posteriorly. The tendon has true synovial sheath. Para tenon with visceral and parietal layers allow for approximately $1.5 \mathrm{~cm}$ of tendon glide.

Blood supply is from musculo tendinous junction, periosteal arterial branches, and meso tenal vessles. Points of importance that are to be noted are

- It is sparse 4-6cms proximal to insertion

- Blood supply decreases with age

- Insertion- to middle inferior $2 / 3^{\text {rd }}$ of calcaneum

\section{Evaluation}

Examination- The points to be noted in examination are as follows,

- palpable defect or prominent scar tissue

- intact but weak active plantar flexion

- increase passive dorsiflexion

- $\quad$ calf atrophy with chronic ruptures 


\author{
Thomson's test \\ Calf squeeze test
}

\title{
III. Managment
}

Controversies still exist on operative and non operative treatment

In acute Achilles tendon rupture - operation are at a higher demand for young athletes'. treatment is preferred for those with limited functional goals or medical co morbidities.

\subsection{Operative treatment:}

- Direct primary repair with end to end repair using bunnel suture with modified Kessler technique or with inter locking suture technique

- Augmented repair a) fascial turn down

b) Plantaris tendon augmentation

c) Peroneus brevis augmentation

- Percutaneous repair

\section{Materials And Methods}

The cases operated during the past one year were studied and 6 months of follow up done till date. Retrospectively medical records reviewed, patients followed up during their post operative visits. The outcome was assessed with Boyden scoring system. Postoperative complications were charted using case sheets study.

\subsection{Operative Technique}

All patients underwent open surgical procedure under spinal anaesthesia in prone position. Longitudinal incision was made $1 \mathrm{~cm}$ medial to Achilles tendon preserving lesser saphenous vein and sural nerve, incision was deepened to full thickness of skin, subcutaneous tissue and paratenon. Ruptured end identified and minimally debrided and modified Kessler repair done with prolene 2-0. Minimum of 2 stiches taken at atleast $2 \mathrm{~cm}$ from the edge. When the distal end was found avulsed along with a piece of calcaneum, steel wire was used. Epitenon and paratenon were repaired with absorbable 3-0 suture. Skin is sutured. After thorough wash, plaster caste applied with 20 degree plantar flexion. Window was created for contaminated wounds for regular care of the wound.

\subsection{Post op instructions}

2 weeks: Cast is removed, suture removed, another short leg cast with foot end gravity equines worn for 2 weeks.

4 weeks-cast changed, foot gradually brought to plantigrade in 2 weeks.

-walking gradually resumed with partial weight bearing with crutches

6-8 weeks (i) short leg walking cast with foot in plantigrade foot

(ii) Full weight bearing allowed

(iii) Active range of motion exercises for 20 min twice daily

(iv) Isometric ankle exercises plus knee and hip strengthening

(v) Toe raises, progressive resistance exercises and proprioceptive exercises

\subsection{Clinical outcome measurement} satisfaction

Boyden score grades clinical factors ${ }^{3}$ such as pain, appearances, footwear restrictions and patient

Excellent - no pain, no limitations in recreational or daily activities. No footwear restrictions and is satisfied with treatment outcome

Good - mild occasional pain, limitations of recreational but not daily activities, no footwear restrictions and satisfied with minor reservations

Fair - mild to moderate pain, limitation of recreational and daily activities. Moderate footwear restrictions and is satisfied but with major reservations

Poor - moderate to severe pain, limitation of recreational and daily activities. Severe footwear restrictions and dissatisfied and has had repeated Achilles tendon re rupture

4.4 Post op complications

-Superficial wound infections

-Deep infections

-Heel ulcer

-Partial Achilles re-rupture 
-Complete Achilles re-rupture

\section{Results}

Ten patients were studied; there were $1(10 \%)$ females and 9 (90\%) males. Age wise distribution was 10-30 yrs were 5(50\%) and 30-60 were 5(50\%). Most common etiology was road traffic accident, $8(80 \%)$ and sports $2(20 \%)$. Open wounds were found in $7(70 \%)$ and closed injury in 3(30\%) of cases. 4(40\%) cases had highly contaminated wounds. $4(40 \%)$ had mildly contaminated wound. There was uneventful recovery in $6(60 \%)$ of the cases with post op complications in 4 cases in which 2 had mild soft tissue infection and one had severe deep tissue infection. Skin flap necrosis in one case after the removal of steel wire which had got infected the underlying deep tissue. One case had wound dehiscence which was re sutured again later. These cases were managed with wound debridement and soft tissue coverage procedure in the form of skin graft or flap cover and appropriate wound care and antibiotic coverage Post operative outcome was assessed with Boyden scoring Good- excellent: 9

Fair - poor: 1

No pain 8

Mild pain 2

No activity limitation 8

Limitation with recreational activity - 1

Limitation with daily activity -1

No difficulty with ambulation- 8

Difficulty with ambulation- 2

\section{Discussion}

Surgical complications following open Achilles tendon repair have been commonly reported in the literature and include difficulty with wound healing, development of wound necrosis and infection. Such complications may secondary to the longitudinal incision commonly used for the surgical approach which traverses an area with poor vascularity ${ }^{2}$. In our study it is found that the implant (steel wire) is not tolerated well and tends to cause deep tissue infections and skin necrosis.

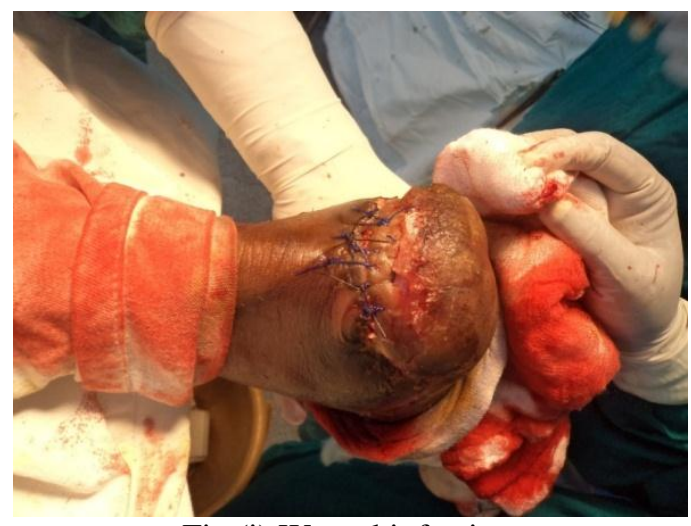

Fig (i) Wound infection

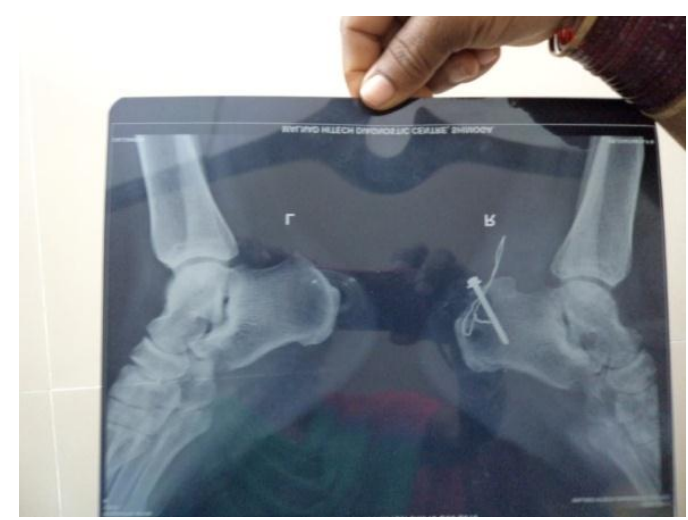

Fig (ii) Wire track infection 


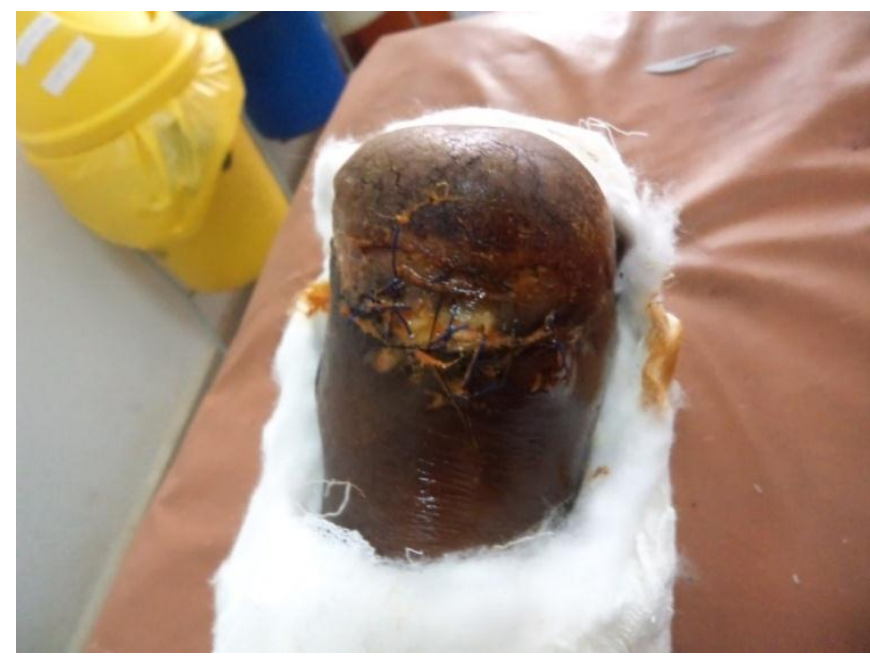

Fig (iii) Skin infection

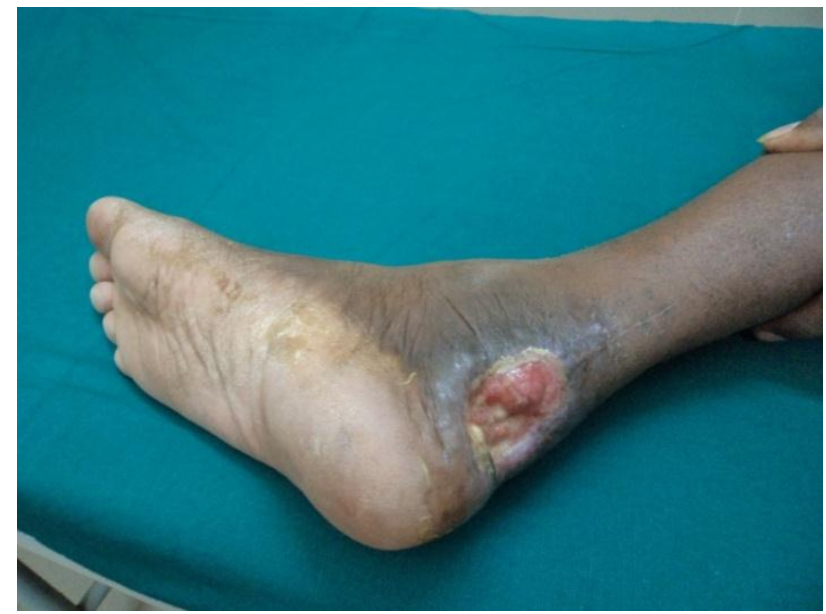

Fig (iv) Flap necrosis

\section{Conclusion}

All the repairs should be handled gently and separation of subcutaneous tissue with peritenon should be in single layer and skin separation from the deeper layers for suturing should never be done.

\section{References}

[1]. Eric J Strauss et all operative treatment of acute Achilles tendon ruptures. An institutional

[2]. review of clinical outcome, injury, int $\mathbf{J}$.care injured (2006)

[3]. DOI: 10.1016/j - injury.2006.06.005

[4]. Haertsch PA. The blood supply to the skin of the leg..A post-mortem investigation Br J Plast Surg $1981 ; 34470-7$

[5]. Boyden EM, Kitoaka HB, Cohalan TD, AnKn. Late v/s early repair of Achilles tendon rupture clinical and biomechanical evaluation. Clin orthop relat res 1995..317:150.8

[6]. O'Bran the anatomy of Achilles tendon foot ankle clin n.AM -2005,10:225-238

[7]. Achilles tendon Wikepedia the free encyclopedia-en.wikipedia.org/wiki/Achilles tendon. 